\title{
OPERATORS ON A QUASI-REFLEXIVE BANACH SPACE
}

\author{
P. M. CUTTLE ${ }^{1}$
}

1. Introduction. Let $X$ be a Banach space, $\mathfrak{B}(X)$ the closure of the algebra of all bounded linear operators on $X$ of finite rank, the closure being taken in the topology of the norm

$$
\|B\|=\sup _{x \in X} \frac{\|B x\|}{\|x\|}, \quad B \in \mathfrak{B}(X) .
$$

The present paper is concerned with a generalization of a theorem of F. Bonsall and A. W. Goldie [1] which states that if $X$ is reflexive then $\mathfrak{B}(X)$ is an annihilator algebra.

It is shown that if $X$ is quasi-reflexive the algebra $\mathfrak{B}(X)$ can be written as the direct sum of four closed subalgebras

$$
\mathfrak{B}(X)=\mathfrak{A}_{1} \oplus \mathfrak{B}_{1} \oplus \mathfrak{B}_{2} \oplus \mathfrak{B}_{3},
$$

where $\mathfrak{A}_{1}$ is a right annihilator algebra which is annihilated on the right by the right ideal $\mathfrak{B}_{2}$ and $\mathfrak{A}_{1} \oplus \mathfrak{B}_{1}$ is a left annihilator algebra which is annihilated on the left by the nilpotent algebra $\mathfrak{B}_{3}$. Moreover if $X$ is reflexive we have $\mathfrak{B}_{1}=\mathfrak{B}_{2}=\mathfrak{B}_{3}=(0)$, so that the above mentioned theorem is obtained as a special case of the present result.

2. Definitions and notation. Let $X$ be a Banach space, $X^{*}$ and $X^{* *}$ its first and second conjugate spaces. The symbol $\pi$ will be used to denote the canonical isomorphism of $X$ into $X^{* *}$. The annihilator in $X^{*}$ of a subspace $Y$ of $X$ will be denoted by $Y^{+}$.

If $x \in X$ and $x^{*} \in X^{*}$ then as in [4] we use the symbol $x \otimes x^{*}$ to denote the one dimensional operator on $X$ defined by the equation

$$
\left(x \otimes x^{*}\right) y=x^{*}(y) x \quad \text { for each } y \in X .
$$

Throughout this paper the closure of the algebra of bounded linear operators of finite rank will be denoted by $\mathfrak{B}(X)$. All algebras of operators under consideration are considered to be normed with the operator bound, that is

$$
\|A\|=\sup _{x \in X} \frac{\|A x\|}{\|x\|} .
$$

If $A$ is an operator on a Banach space $X$, we denote by $A^{*}$ the

Received by the editors August 11, 1961 and, in revised form, October 20, 1961.

1 The author is indebted to the referee for pointing out an error in the original manuscript and for his helpful suggestions with respect to the presentation. 
adjoint of $A$, that is the operator on $X^{*}$ defined by $A^{*} x^{*}(x)=x^{*}(A x)$. The symbol $I$ will denote the identity operator.

Let $\mathfrak{A}$ be an algebra and $\& \subset \mathfrak{A}$. The right (left) annihilator of $\&$ will be denoted by $R(\xi)(L(₹))$.

An algebra $\mathfrak{A}$ is called a right (left) annihilator algebra [4] if for every closed left (right) ideal $\Im$ we have $R(\Im)=(0),(L(\Im)=(0))$ if and only if $\mathfrak{\Im}=\mathfrak{A}$.

A Banach space $X$ is called quasi-reflexive of order $n$ [2] if the quotient space $X^{* *} / \pi X$ is (finite) $n$-dimensional.

\section{Preliminary lemmas.}

3.1. Lemma. Let $X$ be a Banach space, $Y_{i}, i=1,2$, closed linear subspaces of $X$ such that $X=Y_{1} \oplus Y_{2}$. Let $\mathfrak{B}_{i}$ be the subset of $\mathfrak{B}(X)$ consisting of those operators in $\mathfrak{B}(X)$ with range contained in $Y_{i}, i=1,2$. Then $\mathfrak{B}_{i}$ is a closed right ideal in $\mathfrak{B}(X)$ and $\mathfrak{B}(X)=\mathfrak{B}_{1} \oplus \mathfrak{B}_{2}$.

Proof. Let $A \in \mathfrak{B}_{i}, B \in \mathfrak{B}(X)$, then Range $A B \subset Y_{i}$, therefore $\mathfrak{B}_{i}$ is a right ideal. If $A_{n} \in \mathfrak{B}_{i}$ and $A_{n} \rightarrow A$ in the norm topology of $\mathfrak{B}(X)$, then for each $x \in X, A_{n} x \in Y_{i}$ and $A_{n} x \rightarrow A_{x}$ in the norm topology of $X$. Since $Y_{i}$ is closed, $A x \in Y_{i}$, so $A \in \mathfrak{B}_{i}$. Thus, it follows that $\mathfrak{B}_{i}$ is a closed right ideal in $\mathfrak{B}(X)$. Now, since $X=Y_{1} \oplus Y_{2}$ there exists a continuous projection $P$ of $X$ onto $Y_{1}$ with null space $Y_{2}$. If $B \in \mathfrak{B}(X)$ we can write $B=P B+(I-P) B$, where $P B \in \mathfrak{B}_{1}$ and $(I-P) B \in \mathfrak{B}_{2}$. This decomposition is obviously unique and therefore $\mathfrak{B}(X)=\mathfrak{B}_{1} \oplus \mathfrak{B}_{2}$.

Lemma 2. Let $X, Y_{1}, \mathfrak{B}_{1}$ be as in Lemma 1 , let $Z_{i}, i=1,2$ be closed linear subspaces of $X^{*}$ such that $X^{*}=Z_{1} \oplus Z_{2}$. Let $\mathfrak{A}_{i}$ be the subset of $\mathfrak{B}_{1}$ whose elements are the operators in $\mathfrak{B}_{1}$ whose adjoints have range contained in $Z_{i}, i=1,2$. Then $\mathfrak{A}_{i}$ is a closed left ideal in $\mathfrak{B}_{1}$ and $\mathfrak{B}_{1}=\mathfrak{A}_{1} \oplus \mathfrak{H}_{2}$.

Proof. The proof that $\mathfrak{A}_{i}$ is a closed left ideal is similar to the argument in Lemma 1 and hence is omitted. Let $P$ be the continuous projection of $X$ onto $Y_{1}$ with null space $Y_{2}$ and $Q$ the continuous projection of $X^{*}$ onto $Z_{1}$ with null space $Z_{2}$. Note first that $\mathfrak{A}_{i}, i=1,2$, are closed subspaces of $\mathfrak{B}_{1}$ and that if $A_{i} \in \mathfrak{A}_{i}, i=1,2$, we have $\left\|A_{1}\right\|$ $=\left\|Q\left(A_{1}+A_{2}\right)\right\| \leqq\|Q\|\left\|A_{1}+A_{2}\right\|$. It follows from Theorem $2.1[3]$ that $\mathfrak{A}_{1} \oplus \mathfrak{A}_{2}$ is a closed subalgebra of $\mathfrak{B}_{1}$. Next, we show that $\mathfrak{A}_{1} \oplus \mathfrak{A}_{2}$ is dense in $\mathfrak{B}_{1}$. A one dimensional operator in $\mathfrak{B}_{1}$ is of the form $y \otimes x^{*}$ with $y \in Y, x^{*} \in X^{*}$ and can be written $y \otimes x^{*}=y \otimes Q x^{*}+y \otimes(I-Q) x^{*}$, with $y \otimes Q x^{*} \in \mathfrak{A}_{1}$ and $y \otimes(I-Q) x^{*} \in \mathfrak{A}_{2}$. It follows that every operator of finite rank in $\mathfrak{B}_{1}$ can be written as the sum of an operator in $\mathfrak{A}_{1}$ and an operator in $\mathfrak{A}_{2}$. Now, if $B \in \mathfrak{B}_{1} \subset \mathfrak{B}(X)$, there exists a sequence $F_{n}$ of operators of finite rank on $X$ such that $F_{n} \rightarrow B$ in 
the norm topology. Since $P$ is a continuous projection on $Y_{1}$ and Range $B \subset Y_{1}$ we also have $P F_{n} \rightarrow B$ where $P F_{n}$ is an operator of finite rank belonging to $\mathfrak{B}_{1}$ and therefore $P F_{n} \in \mathfrak{A}_{1} \oplus \mathfrak{U}_{2}$. It follows that $\mathfrak{A}_{1} \oplus \mathfrak{A}_{2}$ is dense in $\mathfrak{B}_{1}$ and therefore $\mathfrak{A}_{1} \oplus \mathfrak{A}_{2}=\mathfrak{B}_{1}$.

LemMA 3. If $X$ is quasi-reflexive of order $n$ then there exists Banach spaces $X_{1}$ and $X_{2}$ and an equivalent norm for $X$ such that $X_{2}^{*}=X_{1}$, $X_{1}^{*}=X$ and

$$
\begin{aligned}
& X^{*}=X_{1}^{* *}=\pi_{1} X_{1} \oplus\left(\pi_{2} X_{2}\right)^{+} \\
& X=X_{2}^{* *}=\pi_{2} X_{2} \oplus V^{+} \\
& X_{1}=X_{2}^{*}=V \oplus U
\end{aligned}
$$

where $\pi_{i}$ is the canonical embedding of $X_{i}$ in $X_{i}^{* *}, i=1,2$, and where $U, V^{+}$and $\left(\pi_{2} X_{2}\right)^{+}$are all (finite) $n$-dimensional.

Proof. Since $X$ is quasi-reflexive of order $n$, it follows from Theorem 3.5 of [2] that $X_{1}, X_{2}$ exist such that $X_{2}^{*}=X_{1}$ and $X_{1}^{*}=X$ and such that both $X_{1}$ and $X_{2}$ are quasi-reflexive of order $n$. The direct sum decompositions follow at once from Theorem 3.3 and the proof of Theorem 3.1 of [2].

4. Theorem. Let $X$ be a quasi-reflexive space. Then $\mathfrak{B}(X)$ $=\mathfrak{A}_{1} \oplus \mathfrak{B}_{1} \oplus \mathfrak{B}_{2} \oplus \mathfrak{B}_{3}$ where $\mathfrak{A}_{1}$ is a right annihilator algebra and $\mathfrak{B}_{2}$ a right ideal, which annihilates $\mathfrak{A}_{1}$ on the right $; \mathfrak{A}_{1} \oplus \mathfrak{B}_{1}$ is a left annihilator algebra and $\mathfrak{B}_{3}$ a nilpotent algebra which annihilates $\mathfrak{A}_{1} \oplus \mathfrak{B}_{1}$ on the left. Moreover the following are equivalent:

(a) $\mathfrak{A}_{1}$ is a left annihilator algebra,

(b) $\mathfrak{B}_{i}=(0), i=1,2$, or 3 ,

(c) $X$ is reflexive.

Proof. By Lemma 3 there exist Banach spaces $X_{1}$ and $X_{2}$ such that

$$
X^{*}=\pi_{1} V \oplus \pi_{1} U \oplus\left(\pi_{2} X_{2}\right)^{+} \text {and } X=\pi_{2} X_{2} \oplus V^{+} .
$$

Let $\mathfrak{A}_{1}$ denote the subalgebra of $\mathfrak{B}(X)$ whose elements are the operators in $\mathfrak{B}(X)$ with range in $\pi_{2} X_{2}$ and which have adjoints with range in $\pi_{1} V$; let $\mathfrak{B}_{1}$ denote the subalgebra of $\mathfrak{B}(X)$ whose elements are those operators in $\mathfrak{B}(X)$ with range in $\pi_{2} X_{2}$, which have adjoints with range in $\pi_{1} U$; let $\mathfrak{B}_{2}$ denote the right ideal of $\mathfrak{B}(X)$ whose elements are the operators in $\mathfrak{B}(X)$ with range in $V^{+}$; and let $\mathfrak{B}_{3}$ be the subalgebra of $\mathfrak{B}(X)$ consisting of those operators with range in $\pi_{2} X_{2}$ and whose adjoints have range in $\left(\pi_{2} X_{2}\right)+$. Then an application of Lemma 1 and Lemma 2 yields

$$
\mathfrak{B}(X)=\mathfrak{A}_{1} \oplus \mathfrak{B}_{1} \oplus \mathfrak{B}_{2} \oplus \mathfrak{B}_{3} .
$$


Next we will show that $\mathfrak{A}_{1}$ is a right annihilator algebra. By the proof of Theorem 3.1 [2] we see that there exists in isomorphism $\alpha$ of $\pi_{2} X_{2}$ onto $V^{*}$ such that $\alpha\left(\pi_{2} x_{2}\right)(v)=v\left(x_{2}\right)$ for all $x_{2} \in X_{2}, v \in V$.

We establish next an isomorphism $\beta$ of $\mathfrak{A}_{1}$ onto $(\mathfrak{B}(V))^{*} .^{2}$ For $A \in \mathscr{N}_{1}$ define $\beta A=\alpha A \alpha^{-1}$. If we let $T \in \mathfrak{B}(V)$ be defined by $T v$ $=\pi_{1}^{-1} A^{*} \pi_{1} v$ for all $v \in V$, then

$$
\left(T^{*} v^{*}\right)(v)=v^{*}\left(\pi_{1}^{-1} A^{*} \pi_{1} v\right)=\left(A^{*} \pi_{1} v\right)\left(\alpha^{-1} v^{*}\right)=\left(\alpha A \alpha^{-1} v^{*}\right)(v),
$$

and it follows that $\beta A=T^{*}$ and hence $\beta A \in(\mathfrak{B}(V))^{*}$.

The mapping $\beta$ is onto since if $T^{*} \in(\mathfrak{B}(V))^{*}$ we can define $A \in \mathfrak{B}(X)$ by $A=\alpha^{-1} T^{*} \alpha P$, where $P$ is the continuous projection of $X$ onto $\pi_{2} X_{2}$, with null space $V^{+}$; clearly Range $A \subset \pi_{2} X_{2}$, and $\beta A=T^{*} \alpha P \alpha^{-1}$ $=T^{*}$. To show that Range $A^{*} \subset \pi_{1} V$ we proceed as follows. Suppose first that $T$ is a one-dimensional operator, then $T=v \otimes v^{*}$ for some $v \in V, v^{*} \in V^{*}$, and $T^{*}=v^{*} \otimes \pi v$, where $\pi$ is the canonical embedding of $V$ into $V^{* *}$. We then have $A=\alpha^{-1}\left(v^{*} \otimes \pi v\right) \alpha P$ and for any $x^{*} \in X^{*}$, $x \in X$ we obtain $A^{*} x^{*}(x)=x^{*}\left[\alpha^{-1}\left(v^{*} \otimes \pi v\right) \alpha P x\right]=\pi v(\alpha P x) x^{*}\left(\alpha^{-1} v^{*}\right)$ $=(P x)(v) x^{*}\left(\alpha^{-1} v^{*}\right)$ using the definition of $\alpha$. Now $x=P x+(I-P) x$ and $(I-P) x \in V^{+}$, so $\pi_{1} v(x)=x(v)=P x(v)$, and hence $A^{*} x^{*}$ $=x^{*}\left(\alpha^{-1} v^{*}\right) \pi_{1} v \in \pi_{1} V$. This shows that Range $A^{*} \subset \pi_{1} V$ in case $T$ is a one-dimensional operator. Similarly if $T$ is of finite rank we obtain Range $A^{*} \subset \pi_{1} V$. Finally, if $T$ is arbitrary in $\mathfrak{B}(V)$, there exists a sequence $T_{n}$ of operators of finite rank such that $T_{n} \rightarrow T$. Let $A_{n}$ $=\alpha^{-1} T_{n}^{*} \alpha P$ and $A=\alpha^{-1} T^{*} \alpha P$. Since $T_{n} \rightarrow T$, since $\pi_{1} V$ is a closed subspace of $X^{*}$ and since Range $A_{n}{ }^{*} \subset \pi_{1} V$ for each $n$, it follows that $A_{n} \rightarrow A$ and Range $A^{*} \subset \pi_{1} V$. The mapping $\beta$ is therefore onto $(\mathfrak{B}(V))^{*}$.

It is clear furthermore that $\beta$ is one to one, linear, bicontinuous and preserves multiplication, i.e., $\beta(A B)=(\beta A)(\beta B)$ for all $A, B \in \mathfrak{A}_{1}$. We can thus identify $\mathfrak{A}_{1}$ and $(\mathfrak{B}(V))^{*}$.

Now, $\mathfrak{B}(V)$ is a left annihilator algebra [4, p. 107] so $(\mathfrak{B}(V))^{*}$ is a right annihilator algebra. It follows that $\mathfrak{A}_{1}$ is a right annihilator algebra.

Next, if $A \in \mathfrak{A}_{1}$ and $B \in \mathfrak{B}_{2}$ then for any $x^{*} \in X^{*}$ and $x \in X$ we have $\left((A B)^{*} x^{*}\right) x=\left(A^{*} x^{*}\right)(B x)=0$, since $A^{*} x^{*} \in \pi_{1} V$ and $B x \in V^{+}$. Therefore $(A B)^{*}=0$ so that $A B=0$, hence $\mathfrak{B}_{2}$ annihilates $\mathfrak{A}_{1}$ on the right.

We consider next $\mathfrak{A}_{1} \oplus \mathfrak{B}_{1}$ which is the subalgebra consisting of those operators in $\mathfrak{B}(X)$ with range in $\pi_{2} X_{2}$ and whose adjoints have range in $\pi_{1} X_{1}$. In order to show that $\mathfrak{A}_{1} \oplus \mathfrak{B}_{1}$ is a left annihilator algebra it suffices to notice that $\mathfrak{A}_{1} \oplus \mathfrak{B}_{1}=\left(\mathfrak{B}\left(X_{2}\right)\right)^{* *}$. This equality follows from the fact that if $T \in \mathfrak{B}\left(X_{2}\right)$ and $T=x_{2} \otimes x_{1}$ with $x_{2} \in X_{2}$ and $x_{1} \in X_{1}=X_{2}^{*}$

2 If $\mathfrak{B}$ is a set of operators we denote by $\mathfrak{B}^{*}$ the set $\left\{T^{*} \mid T \in \mathfrak{B}\right\}$. 
then $T^{* *}=\pi_{2} x_{2} \otimes \pi_{1} x_{1} \in \mathfrak{A}_{1} \oplus \mathfrak{B}_{1}$, so that $\mathfrak{A}_{1} \oplus \mathfrak{B}_{1}$ and $\left(\mathfrak{B}\left(X_{2}\right)\right)^{* *}$ contain the same one-dimensional operators.

That $\mathfrak{B}_{3}$ annihilates $\mathfrak{A}_{1} \oplus \mathfrak{B}_{1}$ on the left follows from the fact that if $A \in \mathfrak{A}_{1} \oplus \mathfrak{B}_{1}, \quad B \in \mathfrak{B}_{3} \quad x^{*} \in X^{*}$ and $x \in X$ we have $\left((B A)^{*} x^{*}\right)(x)$ $=\left(B^{*} x^{*}\right)(A x)=0$, since $B^{*} x^{*} \in\left(\pi_{2} X_{2}\right)^{+}$and $A x \in \pi_{2} X_{2}$, so that $(B A)^{*}$ $=0$ and therefore $B A=0$. The same conclusion holds if $A \in \mathfrak{B}_{3}, \mathfrak{B}_{3}$ is therefore nilpotent.

Finally we notice that if $\mathfrak{A}_{1}$ is a left annihilator algebra it is an annihilator algebra and so are $(\mathfrak{B}(V))^{*}$ and $\mathfrak{B}(V)$, consequently by [1] $V$ is a reflexive Banach space and so is $V^{*}$. But $V^{*}$ is isomorphic with $X_{2}$ by proof of Theorem 3.1 [2]; so $X_{2}$ is reflexive and by Lemma 3 we conclude that $X$ is reflexive. If $X$ is reflexive then $U=V^{+}=\left(\pi_{2} X_{2}\right)^{+}$ $=(0)$ which implies $\mathfrak{B}_{i}=(0)$ for $i=1,2$ and 3 .

If $\mathfrak{B}_{i}=(0)$ this implies either $U=(0)$ or $V^{+}=(0)$ or $\left(\pi_{2} X_{2}\right)^{+}=(0)$. But by Lemma 3 either one of these inequalities implies the other two so that $\mathfrak{B}_{1}=(0)$ and $\mathfrak{A}_{1} \oplus \mathfrak{B}_{1}=\mathfrak{A}_{1}$, and hence $\mathfrak{A}_{1}$ is a left annihilator algebra.

\section{BIBLIOGRAPHY}

1. F. Bonsall and A. W. Goldie, Annihilator algebras, Proc. London Math. Soc. 34 (1954), 154-167.

2. P. Civin and B. Yood, Quasi-reflexive spaces, Proc. Amer. Math. Soc. 8 (1957), 906-911.

3. E. R. Lorch, On a calculus of operators in reflexive vector spaces, Trans. Amer. Math. Soc. 45 (1939), 217-234.

4. C. E. Rickart, General theory of Banach Algebras, Van Nostrand, New York, 1960.

The University of Saskatchewan 